\title{
BILINEAR SOCTAL AND BIOLOGICAL CONTROL SYSTEMS
}

by

R. R. Mohler

Department of Electrical \& Computer Engineering

Oregon State University; Corval1is, Oregon 97331

\section{OBJECT}

The purpose of this paper is to:

1. Review briefly some relevant results of bilinear system theory and its socioeconomic application.

2. Develop a bilinear model for the realization of a biological process from experimental tracer data.

\section{INTRODUCTION}

Finite state bilinear systems are described by a system of differential equations which are linear in state, linear in control, but not jointly linear in both as are traditional linear systems. An introduction to the physical relevance of these systems, their controllability and optimal control is analyzed in references [1] and [2]. It is shown that linear system controllability and performance may be improved by the addition of a bilinear control mode. This is as it would be expected since the bilinear system does exhibit a form of adaptive control and variable structure. These are the reasons that bilinear systems, or at least their approximation, are so common in natural and societal processes as well as in engineering.

\section{NEUTRON AND CELL FISSION}

Theoretically, any atomic nucleus may undergo fission if it has a mass which is greater than the sum of masses of particles allowed to exist upon division. This situation yields an unstable nucleus, and its fission consequently liberates energy from the reduced mass in subdivision. An activation energy is normaliy required to deform the original nucleus sufficiently so that the electrostatic repulsion force overcomes the binding force of the nucleons. This is not an unusual phenomenon since chemical combustion processes and biological cell division take place in a similar manner. All of these processes are analogous to liquid-drop division which roughly proceeds through geometrical stages of a sphere, an ellipsoid, a dumbell and finally two spheres by the application of an external force to overcome surface tension. 
The point neutron dynamics from nuclear fission, with neutron population level $n$ proportional to power level, is described by the following bilinear system of equations:

$$
\frac{d n}{d t}=\frac{[u(1-\beta)-1]}{\ell} n-\sum_{i=1}^{6} \lambda_{i} c_{i}
$$

and

$$
\frac{\mathrm{dc}_{i}}{\mathrm{dt}}=\frac{\mathrm{u}_{\mathrm{i}}}{\mathrm{l}} \mathrm{n}-\lambda_{i} \mathrm{c}_{i}, i=1, \ldots, 6
$$

where $\&$ is mean neutron generation time, $c_{i}$ is $i$ th precursor level, $\lambda_{i}$ is ith precursor decay constant, $\beta_{i}$ is the portion of neutrons generated fron the ith precursor with $\sum \beta_{i}=\beta$ and $n$ is neutron multiplication [3]. The control $u$ represents the net evolution of population from generation to generation and must be at least unity for the population to be self perpetuating, i.e., a chain reaction in nuclear fission.

Again, the dynamics of cell population is analogous with celis dying, cells generated from precursors and prompt new cells generated a11 in a controlled manner. While both processes involve variable structure control feedback terms (in some cases bilinear), the basic control mechanisms are considerably different. In a nuclear reactor, for example, inherent temperature feedback alters neutron multiplication or fission effectiveness, and external feedback control loops of a conventional or optimal design may be synthesized [3]. Cell processes, on the other hand, are controlled according to genetic code passed along successive cell generations, according to the cell's function in the synthesis or the maintenance of an organ and according to the cell's environmental conditions. Obviously, there is communication between cells in this controlled process. (It appears that the immune response mechanism utilizes T-cells to establish communication lines with alien cells and then breaks these lines leaving holes in the cellular membranes through which low molecular weight molecules pass out and water rushes in to burst the invader.)

In biology, cells, or combinations of cells, forming organs or functional units within organs may form natural compartmental structures to describe the dynamics of many important transfer processes.

\section{COMPARTMENTAL MODELS IN ENGINEERING AND SOCIETY}

It is found that a compartmental structure arises for processes in engineering and society as we,ll as biology. In a nuclear reactor, for example, the 
dynamics are distributed and nearly bilinear, but ordinary bilinear differential equations (1) and (2) or difference equations are usually used to describe the behavior in different spatial compartments. These may include compartmentation according to such regions as fuel-loaded core, moderated core, homogeneous core, reflector, support structure, control and heat exchange. Neutrons and heat are generated and absorbed in certain compartments and transferred between compartments in the form of flux. Similar transfer mechanisms take place for mass transport, chemical reaction, migration of biological species and numerous generalizations of such processes.

Frequently, the dynamical equations are derived by a balance of energy, mass or force. Suppose a single quantity or substance $x$ is distributed within and moves among $\mathrm{n}$ compartments according to conservation equations of the form

$$
\frac{\mathrm{dx}_{\mathrm{i}}}{\mathrm{dt}}=\sum_{j=1}^{\mathrm{n}} \phi_{i j}-\sum_{k=1}^{n} \phi_{k i}+\phi_{i a}-\phi_{a i}+p_{i}-d_{i}, i=1, \ldots, n,
$$

where $x_{i}$ a generalized state variable may be the amount of substance $x$ in the ith compartment, $\phi_{i j}$ is the flux of $x$ from the $j$ th to the $i$ th compartment, $\phi_{i a}\left(\phi_{a i}\right)$ is the flux of $X$ from (to) the environment to (from) the ith compartment, $p_{i}$ is the rate of production, and $d_{i}$ is the rate of destruction of $X$ in the ith compartment. The primed summation denotes deletion of the ith term.

Assume that compartmental volumes or capacities are constant and that $\mathrm{X}$ moves between compartments and between the system and its environment by simple diffusion. Also, assume that $x$ is at uniform concentration $c_{a}$ in the environment. Then, the system fluxes are described by

$$
\begin{aligned}
& \phi_{i j}=\rho_{i j} x_{j}, \\
& \phi_{a i}=\rho_{a i} x_{i}, \\
& \phi_{i a}=\rho_{i a} c_{a}, i, j=1, \ldots, n, i \neq j,
\end{aligned}
$$

and

where the coefficients $\rho_{i j}$ and $\rho_{a i}$ are exchange parameters which may be constant or may be multiplicative controls. Additive control may be present through the net production of substance $\left(p_{i}-d_{i}\right)$ or through $\rho_{i a} c_{a}$.

It is readily seen from Equations (3) and (4) that a collection of terms leads to a bilinear system of the following form: 


$$
\frac{\mathrm{dx}}{\mathrm{dt}}=\mathrm{Ax}+\sum_{\mathrm{k}-1}^{\mathrm{m}} \mathrm{B}_{\mathrm{k}} \mathrm{u}_{\mathrm{k}} \mathrm{x}+\mathrm{Cu}+\mathrm{y},
$$

where $x \in R^{n}$ is the state vector, $u \in R^{m}$ is the control vector, $A, B_{k}$, and $C$ are $n \times n$, $n \times n$, and $n \times m$ matrices respectively, and $y \in R^{n}$ is a constant vector [1]. Again, $x$ is composed of compartmental quantities of substance $x$; additive control may arise from net production or adjustable environmental influx, and multiplicative control from those intercompartmental and environmental efflux exchange parameters which are manipulated. Additive and multiplicative controls may or may not be independent variables for a particular bilinear system. When they are independent, zeros appear in the appropriate positions of $B_{k}$ and $\mathrm{C}$ where necessary. Those exchange parameters which cannot be manipulated result in the constant coefficients of the $A$ matrix and of vector $y$. While the process may include transport of more than one substance, no generality is lost in utilizing the above form of the equations when there is no coupling of substance fluxes.

Socioeconomic compartments may take the form of geographical regions partitioned according to administrative units or according to land topology. In particular, the former is quite convenient since demographic data, business transactions, etc. are readily available, and necessary surveys can be made within the organizational structure. Here, for example, a multiplicative control variable may be migration rate manipulated by means of attractiveness multipliers such as availability of jobs, housing, transportation, etc.

Compartments also may appear in a non-spatial manner. For demographic components, income levels or household sizes may designate compartments, with transfer between compartments by means of birth, death, or job change. In economics, compartments may separate producers from consumers with the appropriate interflux of capital, products, fuels, materials, services and labor. The mathematical model may take an adaptive form based on supply and demand forces for a market economy or based on government goals for a command economy.

\section{TRACER METHODOLOGY}

For many biological processes and certain socioeconomic processes it is impossible or at least very difficult to measure the necessary inputs and outputs to estimate compartmental system parameters. Sometimes, a tracer or state label may be observed, and the system parameters arrived at from the tracer dynamics [4].

If it is assumed that the tracer in every compartment is distributed uniformIy through substance $x$, that labeled and unlabeled substances behave identically, and that the tracer does not appreciably affect the compartmental system behavior, 
then the tracer system is described by

$$
\begin{aligned}
\frac{d\left(x_{i} a_{i}\right)}{d t} & =\sum_{j=1}^{n} \phi_{i j}(t) a_{j}-\sum_{k=1}^{n} \phi_{k i}(t) a_{i}+\phi_{i a}(t) a_{i a}-\phi_{a i}(t) a_{i}+p_{i}(t) a_{i} \\
& -d_{i}(t) a_{i}+f_{i}(t), \quad i=1, \ldots, n,
\end{aligned}
$$

where $a_{i}(t)$ is the specific activity (fraction of $x$ which is labeled) in compartment $i, a_{i a}(t)$ is the specific activity of substance in influx $\phi_{i a}(t)$, and $f_{j}(t)$ is the influx of tracer which is inserted directly into compartment $i$. Equation ( 6 ) includes, for example, the possibility both of direct tracer insertion by injection into compartments and of tracer uptake by natural flux routes from a labeled environment.

\section{PHYSIOLOGICAL WATER BALANCE}

A two-compartment, water balance model is presented here. Experimental data is used from the so-called wild house mouse in a controlled environment. The plasma compartment contains all the body water, $w_{1}(t)$, in equilibrium with the blood plasma. Similarly, the second compartment or "evaporate" compartment, contains the body water, $w_{2}(t)$, in equilibrium with insensible evaporation from the animal $\left[w_{1}(t)+w_{2}(t)=w_{b}(t)\right.$ total water weight $]$. The plasma compartment receives water from the environment, $\phi_{g}(t)$, via the gastro-intestinal tract in the form of drinking water, as well as hygroscopic and metabolic water from the animal's food. Water is last to the environment from the plasma compartment in the urine, $\phi_{u}(t)$. The evaporate compartment exchanges water with the environment via efflux $\phi_{e^{(t)}}$ and influx $\phi_{a}(t)$. The two compartments, of course, exchange water inside the animal, with flux $\phi_{i j}(t)$ to compartment $i$ from compartment $j$.

By inspection, the water conservation equations for this model are

$$
\begin{aligned}
& \dot{w}_{1}=-\phi_{21}(t)+\phi_{12}(t)-\phi_{u}(t)+\phi_{g}(t) \\
& \dot{w}_{2}=\phi_{21}(t)-\phi_{12}(t)-\phi_{e}(t)+\phi_{a}(t),
\end{aligned}
$$

and in water balance $\left(\dot{w}_{1}=\dot{w}_{2}=0\right)$,

$$
\phi_{21}(t)-\phi_{12}(t)=\phi_{g}(t)-\phi_{u}(t)=\phi_{e}(t)-\phi_{a}(t) .
$$

Since the system is closed and conservative, the associated specific activity kinetics for an ideal tracer introduced into the system (6) by natural flux routes are given by 


$$
S(t)=\left[\begin{array}{rr}
a=S(t) a, & \frac{\phi_{12}(t)}{w_{1}(t)} \\
-\frac{\left.\phi_{12}(t)+\phi_{g}(t)\right]}{w_{1}(t)} & \frac{\left[\phi_{21}(t)+\phi_{a}(t)\right]}{\phi_{21}(t)}
\end{array}\right]
$$

and $a \varepsilon R^{2}$. It can be shown that original system state can be obtained by an adjoint equation of (9).

Also, it can be shown from Gersgorin's first matrix theorem [5] that the eigenvalues are real and distinct in this case. By measurement of the eating and drinking water influx, the urinary and fecal efflux, total body water (estimated from body weight), and the tracer eigenvalues, it is possible to obtain the water in each compartment and the water exchanges with the environment and between compartments.

The measured and unknown quantities for the two-compartment equilibrium model are summarized in Table 1 . The unknown parameters, in turn, are related to the measured quantities by the equations which follow.

TABLE I

Parameters for the two-compartment water model.

$$
\begin{aligned}
& \text { Measured } \quad \phi_{\mathrm{g}} \phi_{\mathrm{u}} \mathrm{w}_{\mathrm{b}} \sigma_{1} \sigma_{2} \mathrm{~m}_{1} \\
& \text { Unknown } \quad \phi_{21} \phi_{12} \phi_{\mathrm{e}} \phi_{\mathrm{a}} \mathrm{w}_{1} \mathrm{w}_{2} \\
& \phi_{21}-\phi_{12}=\phi_{\mathrm{g}}-\phi_{\mathrm{u}} \\
& \phi_{e}-\phi_{a}=\phi_{g}-\phi_{u} \\
& w_{b}=w_{1}+w_{2} \\
& w_{1} w_{2} \sigma_{i}^{2}+\left[\left(\phi_{21}+\phi_{a}\right) w_{1}+\left(\phi_{21}+\phi_{u}\right) w_{2}\right] \sigma_{i}+\phi_{21} \phi_{a}+\phi_{21} \phi_{g} \\
& +\phi_{u} \phi_{a}=0, i=1,2 \\
& m_{1}=\frac{\phi_{21}}{\phi_{21}+\phi_{a}+w_{2} \sigma_{1}} \text {. }
\end{aligned}
$$

From the measured parameters shown in Table II for two equilibrium washout test conditions, a simulation was made of the two compartmental equations with 
the corresponding parameters shown in Table III. The eigenvalues and eigenvector slopes may be used to check the accuracy of the model with the measurements made.

TABLE II

Measured Parameters for Ad Libitum and 1/8 Ad Libitum Drinking Water Conditions.

\begin{tabular}{lcc} 
& Ad Libitum & 1/8 Ad Libitum \\
\cline { 2 - 3 }$\phi_{g}(g /$ day $)$ & 5.0 & 1.9 \\
$\phi_{\mathrm{u}}(\mathrm{g} /$ day $)$ & 2.3 & 1.2 \\
$w_{\mathrm{b}}(\mathrm{g})$ & 14.5 & 11.6 \\
$\sigma_{1}(\cdot /$ day $)$ & -0.3 & -0.2 \\
$\sigma_{2}(\cdot /$ day $)$ & -13 &..- \\
$\mathrm{m}_{1}$ & 0.9 & 0.7
\end{tabular}

TABLE II I

Parameters for Simulations of Ad Libitum and $1 / 8$ Ad Libitum Drinking Water Conditions.

\begin{tabular}{lcc} 
& Ad Libitum & $\frac{1 / 8 \text { Ad Libitum }}{2}$ \\
\cline { 2 - 2 }$\phi_{21}(\mathrm{~g} /$ day $)$ & 10.0 & 2.0 \\
${ }_{12}(\mathrm{~g} /$ day $)$ & 7.3 & 1.3 \\
$\phi_{\mathrm{e}}(\mathrm{g} /$ day $)$ & 4.0 & 1.5 \\
$\phi_{\mathrm{a}}(\mathrm{g} /$ day $)$ & 1.3 & 0.8 \\
$\mathrm{w}_{1}(\mathrm{~g})$ & 13.2 & 10.5 \\
$\mathrm{w}_{2}(\mathrm{~g})$ & 1.3 & 1.1 \\
$\phi_{1}(\cdot /$ day $)$ & -0.35 & -0.19 \\
$\phi_{2}(\cdot /$ day $)$ & -9.2 & -2.7 \\
$\mathrm{~m}_{1}$ & 0.91 & 0.73 \\
\hline
\end{tabular}

Mohler [1] shows that the water excretion in urine, $\phi_{u}$, and body watex, $w_{b}$ may then be related to the control variable, $u_{k}$, by a bilinear equation of the form

$$
\phi_{u}=u_{k}\left(c_{g}+b_{g} w_{b}\right)
$$

where $u_{k}$ is a combination of complicated control processes by means of manipulated permeability, arteriolar resistance and osmotic water pressure in the 
kidneys. Other measurements would have to be made to arrive at estimates of basic physiological parameters such as arteriolar resistance or membrane permeability, and more basic constants than $c_{g}$ and $b_{g}$.

A preliminary analysis of identification in medical diagnosis for processes of this class is presented by Cohen [6].

\section{ACKNOWLEDGEMENT}

The author wishes to acknowledge the contributions of W. D. Smith and the support of the National Science Foundation for this work.

\section{REFERENCES}

1. Mohler, R, R., Bilinear Control Processes, Academic Press, New York and London, 1973.

2. Mohler, R. R. and Ruberti, A., Eds., Theory and Applications of Variable Structure Systems, Academic Press, New York and London, 1972.

3. Mohler, R. R. and Shen, C. N., Optimal Control of Nuclear Reactors, Academic Press, New York and London, 1970.

4. Sheppard, C. W. and Householder, A. S., "The Mathematical Basis of the Interpretation of Tracer Experiments in Closed Steady-State Systems", J. Applied Physics, 22 (1951), 510-520.

5. Lancaster, Peter, Theory of Matrices, Academic Press, New York and London, 1969.

6. Cohen, A., "Parameter Estimation for Medical Diagnosis", Proc. IFAC Identification Conference, The Hague, (1973), 239-241. 